\title{
Cholecystokinin Effects on Feeding, Glucose, and Pancreatic Hormones in Rhesus Monkeys ${ }^{1}$
}

\author{
BONNIE L. METZGER AND BARBARA C. HANSEN ${ }^{2}$ \\ Department of Physiology and the Schools of Medicine and Nursing \\ The University of Michigan, Ann Arbor, MI 48109
}

Received 16 August 1982

\begin{abstract}
METZGER, B. L. AND B. C. HANSEN. Cholecystokinin effects on feeding, glucose, and pancreatic hormones in rhesus monkeys. PHYSIOL BEHAV 30(4) 509-518, 1983.-The effects of cholecystokinin on feeding, and on plasma glucose and pancreatic hormone responses to a mixed-meal were determined in lean rhesus monkeys. Following an overnight fast the octapeptide of cholecystokinin (CCK-8) was administered intravenously over a 6 minute period coincident with the initiation of free feeding or an intragastric infusion of a mixed liquid diet. CCK-8 inhibited feeding and delayed the plasma glucose and insulin response to a mixed-meal. The threshold for the feeding effect ranged from 30 to $120 \mathrm{ng} / \mathrm{kg} / \mathrm{min}$ across monkeys and did not extend beyond 15 minutes of the start of the CCK infusion. The delays in plasma glucose and insulin were not dependent on rate or amount of food entering the stomach. Further, there were no alterations from basal levels in plasma glucose or insulin prior to the onset of CCK-induced feeding inhibition. There was no evidence that CCK-8 stimulated insulin release, nor was the usual close relationship between plasma glucose and insulin levels in response to a mixed-meal changed by CCK-8.
\end{abstract}

Cholecystokinin (CCK-8) Feeding behavior Meal size Meal length Plasma glucose Plasma insulin Plasma glucagon Plasma pancreatic polypeptide Mixed-meal challenge

Rhesus monkeys (Macaca mulatta)

PERIPHERAL intravenous infusions of the exogenous synthetic octapeptide of the brain-gut peptide, cholecystokinin (CCK-8) have been shown to suppress feeding in many animal species, including man $[1,3,6,9,11,26]$. In general, this reduction in intake is food-specific and free of apparent clinical and sub-clinical distress $[1,3,6,11,12]$. The degree of feeding suppression appears to be dependent upon multiple factors, including the amount of CCK infused and the length of the infusion, as well as the length of the food deprivation period preceding each experiment $[21,26]$. With few exceptions, the nature of the CCK-induced feeding effect has been described as a rapid, but limited decrease in the length of the meal $[1,6,9,11]$. In hungry pigs, Anika, Houpt and Houpt demonstrated a rapid threshold type on-off interruption of a meal [1]. CCK did not alter the initial feeding rate in rats or man $[9,11,21]$.

The present study examined the effect of short duration intravenous infusions of $\mathrm{CCK}-8$, at varying doses, on the feeding behavior of rhesus monkeys using a feeding system which allows precise monitoring of food intake and pattern.

We also sought further clarification of CCK-8's effects on the endocrine pancreas. Jensen and associates [10] were unable to stimulate insulin or glucagon release from an isolated pancreas preparation with $\mathrm{CCK}-8$ infused within probable physiological levels. On the other hand, Rouiller and others [23] using intact, anesthetized dogs, administered intravenous CCK -8 and observed increased pancreatic vein and inferior vena cava levels of plasma insulin and glucagon. An indirect role of CCK could be associated with the pre- and post-absorption alterations in the plasma levels of glucose and insulin which have been purported to play significant roles in moment to moment appetite regulation [14]. In addition to the reported effects on plasma glucose and insulin, exogenous CCK-8 has been found to significantly elevate pancreatic polypeptide levels [15]. Further, McLaughlin and Baile [17] have demonstrated that intraperitoneal injections of the pancreatic polypeptide decreased food intake in lean mice.

The present experiments in monkeys examined the effects of intravenous CCK-8 on the levels of plasma glucose, insulin, glucagon, and pancreatic polypeptide in response to an oral or intragastrically infused mixed meal. Furthermore,

'This investigation was supported in part by a Department of Health and Human Services, U.S.P.H.S. National Research Service Award (NU-05338) from the Division of Nursing, the National Institutes of Health (AM-20493) and AM-20572 (Michigan Diabetes Research and Training Center), the Northwest Medical Research Foundation and Warner Lambert-Parke Davis Pharmaceutical Research Division.

${ }^{2}$ Requests for reprints should be addressed to B. C. Hansen, Ph.D., Associate Vice President for Research and Dean of the Graduate School, Southern Illinois University at Carbondale, Carbondale, IL 62901. 
these experiments sought to identify any association between effects of CCK-8 on feeding behavior and the changes across time in mixed-meal stimulated plasma levels of glucose and pancreatic hormones.

\section{METHOD}

Five young adult male rhesus monkeys (Macaca mulatta) weighing from six to nine $\mathrm{kg}$ were studied. The monkeys were accustomed to three-tiered primate restraint chairs and were housed in a temperature controlled room $\left(22^{\circ} \mathrm{C}\right)$ with a 12-hour light/dark cycle (lights on at $0700 \mathrm{hr}$ ). Each monkey was accustomed to an eight hour/day feeding regimen $(0800$ to 1600 hours), and a completely balanced liquid diet (Ensure ${ }^{\odot}$, Ross Laboratories) which contains $1.06 \mathrm{kcal} / \mathrm{ml}$ with $14 \%$ of the calories as protein, $31.5 \%$ fat, and $54.5 \%$ carbohydrate. The liquid diet was delivered via a suckingactivated automated feeding system designed to provide each monkey with free access to food during the daily eight hour feeding period, while at the same time permitting microcomputer recording of detailed patterns of ingestion [25], including meal size (kcal), meal duration (seconds), and inter-meal interval (minutes). Each meal was defined as a period of sustained feeding (minimum amount $20 \mathrm{kcal}$ ) separated from the next such period by at least five minutes, and the period of separation was defined as the inter-meal interval (IMI). Water was available ad lib, $24 \mathrm{hr} /$ day.

Following adaptation to the laboratory, restraint, liquid diet and feeding system, each monkey was surgically implanted with silastic intravenous and intragastric cannulas. The intravenous cannulas ( $1 \mathrm{~mm}$ ID, $2.2 \mathrm{~mm}$ OD) were inserted into the internal or external jugular veins, and passed so that the tips were located in the superior vena cava, just above the atrium, or were inserted via the femoral veins to the inferior vena cava. Two intravenous cannulas were used to allow simultaneous infusion and withdrawal. The cannulas were kept patent by continuous infusion of heparinized isotonic saline $(4 \mathrm{U} / \mathrm{ml})$ at a rate between $25-50 \mathrm{ml} / 24$ hour period. An intragastric cannula (1.2 $\mathrm{mm}$ ID, $2.4 \mathrm{~mm}$ OD) was placed in the body of the stomach. Following surgery, all experiments were delayed until the animal resumed feeding at levels approximating those preceding surgery.

In all experiments the synthetic sulphated C-terminal octapeptide of cholecystokinin (CCK-8) (Kinevac, Squibb) was mixed with distilled water to a concentration of $1 \mu \mathrm{g} / \mathrm{ml}$. Each experimental dose was mixed with an isotonic saline carrier to equalize the volumes infused, and the flow rate of a Harvard pump was adjusted to infuse the solution at a constant rate of $4.75 \mathrm{ml} / \mathrm{min}$. Saline controls of equal volume were infused at the same rate. Each infusion lasted exactly six minutes. CCK doses ranged from 15 to $120 \mathrm{ng} / \mathrm{kg} / \mathrm{min}$, and were administered in random order, with all experiments conducted on separate days

The plasma glucose, insulin (IRI), glucagon (IRG) and pancreatic polypeptide (PP) responses to six minute intravenous (IV) infusions of CCK-8, administered simultaneously with either the initiation of free feeding or the initiation of an intragastric mixed-meal infusion, were determined. The time courses of these responses were followed from immediately prior to the start of the CCK-8 infusion (following a 16-hour overnight fast) to 120 minutes. Blood samples were withdrawn immediately before and at intervals of $5,10,20,30$, 40,60 and $120 \mathrm{~min}$ from the start of the mixed meal challenge and IV infusion of CCK. Two milliliters of a mixture of blood and heparin solution were withdrawn to clear the cannula dead space followed by the removal of blood samples of three $\mathrm{ml}$ each. The dead space fluid was returned to the animal after each sample was withdrawn. Blood samples were placed in ice in sterile heparinized tubes and centrifuged at 2000 RPM for six minutes, within 20 minutes of the draw. The plasma was aliquoted into appropriate assay and storage tubes and frozen at $-20^{\circ} \mathrm{C}$. The residual blood cells were maintained in sterile containers, reconstituted with bacteriostatic saline and returned to the monkey at the completion of each study. Plasma levels of glucose were measured by the hexokinase and glucose-6-phosphate dehydrogenase method of Slein [24]. Double-antibody radioimmunoassays were used to determine plasma IRI [8], IRG [22] and PP [4] levels.

Monkeys were not disturbed during the sample collection period and were observed systematically every three minutes for three seconds through a one-way mirror during the first 45 minutes of the experimental period. Behavioral rating scales were used to record these observations, and included sleeping, resting, drinking, eating, sickness behavior and non-ingestive activity. Sleeping behavior was defined as no observable movement, head back and relaxed, and eyes closed; resting as no observable movement, head back and eyes partially closed; feeding as sucking or licking food spout; drinking as licking or sucking water spout; sickness behavior as restlessness, irritability, retching or vomiting; and non-ingestive activity as any movement of the body, arms, hands, head or eyes not related to feeding, drinking or sickness behavior.

In the first set of experiments, the six minute infusion of CCK-8 was started simultaneously with the start of voluntary feeding of the first meal following the usual 16-hour overnight fast. Feeding behavior and plasma glucose, IRI, IRG and PP levels were monitored. In order to examine the CCK-8-induced changes in plasma levels of glucose and pancreatic hormones following a mixed meal independent of the effects of CCK-8 on feeding behavior, a second series of experiments used controlled intragastric infusions of the same liquid diet as was taken orally in the first experiments. The liquid diet was infused at a rate of $10 \mathrm{ml} / \mathrm{min}$ for $10 \mathrm{~min}$ (100 kcal total), and plasma levels of glucose, IRI, IRG, and PP were determined at intervals up to $120 \mathrm{~min}$. Consistency between the oral and intragastric studies was maintained by initiating the intragastric infusion of the mixed-meal challenge concurrently with the start of the intravenous infusion of CCK or saline immediately at the end of the monkey's accustomed 16-hour overnight fast.

A univariate, one-sample $t$-test which allows the testing of the mean of a single sample of observations against an a priori value, (in this case baseline control feeding behavior) was used to describe the within-monkey differences in feeding behavior associated with CCK-8 infusion. For descriptive purposes each feeding parameter (meal size, meal duration. IMI length and total daily intake) for each animal, and CCK experiment, was substituted for the a priori value and compared to a control mean derived from a sampling of the same animal's usual daily eating data ( $N=9$ days). One way analysis of variance (ANOVA) was used to estimate the across monkey differences in feeding behavior as a function of CCK infusions. When significant experimental effects occurred, Scheffe's contrast method was used to identify the specific ways in which the experimental effects contributed to the overall F-ratio [7]. This procedure included the use of protected paired $t$-tests with a 0.95 Scheffe allowance which compensate for the inflated alpha associated with multiple 
$t$-tests. Only the results of the protected $t$-tests are reported unless otherwise noted. Protected paired $t$-tests were also used as a basis for describing more specific effects of CCK on plasma glucose, IRI, IRG and PP. Because there were significant between-animal differences in basal levels, the basal level of each peptide or substrate was subtracted from each subsequent value collected over the 120-minute sampling period, thus identifying the total increment above basal levels for each parameter at each time point. Total response area for each parameter from 0 to $120 \mathrm{~min}$ was also calculated and the above mentioned analysis procedure using ANOVA and protected paired $t$-tests was used to identify differences in response areas compared to saline controls.

Glucose and insulin values were standardized to permit further analysis of changes in the insulin/glucose ratio across time and across various CCK- 8 doses. The significances of differences in these ratios between $\mathrm{CCK}$ doses across animals were determined for each time point by protected paired $t$-tests. Finally, the initial overall within-animal glucose, insulin, glucagon and pancreatic polypeptide response to the mixed meal stimulus across experimental conditions was determined by profile analysis techniques. Because the obvious differences in the hormones and glucose responses occurred within the first $\mathbf{4 0}$ minutes of the experiment, analysis focused on the time period 0-40 minutes. Profile analysis allows the separate testing of (a) the differences between the overall plasma response curves across time for all 4 doses of CCK and saline controls, (b) the differences in the shapes of the response curves (parallelism), and (c) the hypothesis that the curves are flat (plasma levels do not change across time) [7].

\section{RESULTS}

\section{CCK-8 Effect on Feeding Behavior}

CCK-8, administered intravenously during the first six minutes of a meal following an overnight fast, has an immediate and significant effect on intrameal pattern at the lowest doses tested $(30 \mathrm{ng} / \mathrm{kg} / \mathrm{min})$ and temporarily causes cessation of feeding at the highest dose $(120 \mathrm{ng} / \mathrm{kg} / \mathrm{min})$ in all monkeys studied. The minimal six minute infusion dose required to terminate an ongoing meal following an overnight fast differed between monkeys, ranging from 30 to 120 $\mathrm{ng} / \mathrm{kg} / \mathrm{min}$.

As shown in Fig. 1, the usual size of the first meal following an overnight fast in these monkeys ranged from 227 to $281 \mathrm{kcal}$. The infusion of CCK-8 at a dose of $30 \mathrm{ng} / \mathrm{kg} / \mathrm{min}$ had no effect on meal size in 3 of 4 monkeys, and maximal suppressive effect in the fourth monkey. An infusion level of $60 \mathrm{ng} / \mathrm{kg} / \mathrm{min}$ produced near maximal effects on meal size in all but one monkey; the latter required $120 \mathrm{ng} / \mathrm{kg} / \mathrm{min}$ to produce the cessation of ingestion during the course of a meal. Further, all animals stopped eating within 2 minutes when CCK was infused at a rate of $120 \mathrm{ng} / \mathrm{kg} / \mathrm{min}$. In general, meal sizes below $60 \mathrm{kcal}$ reflect the intake during the first $1-2$ min of the availability of food. Since CCK-8 infusions were begun simultaneously with the onset of feeding, the CCK effect was not apparent in truncating the ongoing meal until 1-2 min into the feeding period. Thus, for all monkeys, the minimal dose which affected meal size produced a maximal or near maximal reduction in meal size, indicating the abrupt onset of the CCK effect.

Reduction in the duration of the test meal was more sensitive to the effect of $\mathrm{CCK}$, with significant decreases in meal length observed usually at 30 and always at $60 \mathrm{ng} / \mathrm{kg} / \mathrm{min}$;
COMPARISON OF FIRST MEAL SIZE DURING

IV CCK INFUSION TO CONTROLS $(\bar{x} \pm S E)$

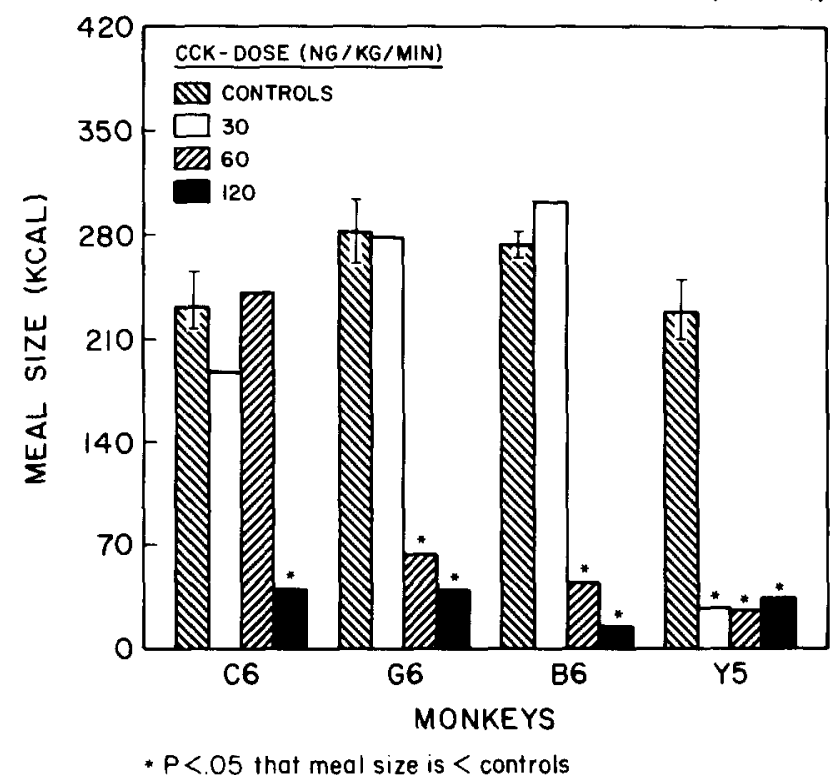

FIG. 1. Effect of a $6 \mathrm{~min}$ intravenous infusion of CCK-8 at three dose levels on meal size $(\mathrm{kcal})$, compared to control or saline infusion conditions following an overnight fast.

further increase in dose resulted in even greater reduction in meal duration: $t(16)=-4.9, p<0.001$ (Table 1).

The cumulative intakes for one monkey (B-6) at each of three doses of CCK-8 and under saline control conditions are shown in Fig. 2A. Both changes in first meal size and in pattern of feeding are apparent at doses of 60 and 120 $\mathrm{ng} / \mathrm{kg} / \mathrm{min}$. It is apparent from this figure for a single representative animal, that the rate of feeding was not affected within any single feeding bout, but longer periods of rest or interruptions in feeding were clearly observed. Furthermore, when early interruptions of feeding occurred, the amount taken during the first 40 minutes was reduced (Fig. 2A).

Across monkeys when a meal was either terminated or reduced by CCK-8 infusion, the subsequent inter-meal interval (IMI) was very short-less than $35 \%$ of the normal IMI duration for each monkey, or an actual IMI of only 6 to 20 min compared to the usual control IMI (mean $\pm S E$ ) of $155 \pm 16 \mathrm{~min}$ (Table 1). The amount ingested in the meal immediately following the CCK-8-induced interruption of feeding, when added to the initial feeding amount prior to the onset of the CCK effect, $218 \pm 15 \mathrm{kcal}$ (mean $\pm \mathrm{SE}$ ), generally approximated the total consumed in the usual first meal under control conditions $(251 \pm 14 \mathrm{kcal}): t(8)=-2.2, p=\mathrm{ns}$. Within the first three hours following the six minute CCK-8 infusion, most animals initiated a second meal, as was their usual pattern, and the food intake had returned to within normal levels for that time period (control $=326 \pm 16 \mathrm{kcal}$ as compared to $300 \pm 17 \mathrm{kcal}$ ), thus reinforcing the finding of an extremely transient effect of intravenous CCK-8. Total day's intake, and the intake of the subsequent day showed no effect of the short-term CCK-8 infusion compared to saline controls.

In summary, the dose of CCK-8, over a range of 30 to 120 $\mathrm{ng} / \mathrm{kg} / \mathrm{min}$ infused for a six minute period was negatively 
TABLE 1

EFFECTS OF VARYING DOSES OF CCK-8 ON THE SIZE OF THE FIRST MEAL, ITS DURATION, THE RATE OF INTRA-MEAL FEEDING, AND THE DURATION OF THE CCK INDUCED INTERRUPTION IN FEEDING

\begin{tabular}{|c|c|c|c|c|c|}
\hline Monkey & $\underset{\text { (ng/kg/min) }}{{ }^{*} \text { CCK-8 Dose }}$ & $\begin{array}{c}\text { Size of } \\
\text { first meal } \\
(\% \text { of control })\end{array}$ & $\begin{array}{l}\text { Length of } \\
\text { first meal } \\
\text { (\% of control) }\end{array}$ & $\begin{array}{c}\text { Feeding rate } \\
\text { during first meal } \\
(\mathrm{ml} / \mathrm{sec})\end{array}$ & $\begin{array}{c}\text { Duration of } \\
\text { first inter-meal } \\
\text { interval } \\
\text { (\% of control) }\end{array}$ \\
\hline B-6 & 120 & $5^{\dagger}$ & $3+$ & 0.44 & $6^{\dagger}$ \\
\hline$C-6$ & 120 & $17 \dagger$ & $9+$ & 0.33 & $10^{\dagger}$ \\
\hline G-6 & 120 & $21 \dagger$ & $10^{\dagger}$ & 0.56 & $11 \div$ \\
\hline$Y-5$ & 120 & $14 \dagger$ & $8+$ & 0.46 & $34 \dagger$ \\
\hline mean $\pm S E$ & - & $14 \pm 3 \%$ & $8 \pm 2 \%$ & $0.45 \pm 0.05$ & $16 \pm 06 \%$ \\
\hline B-6 & 60 & $16+$ & $23^{\dagger}$ & 0.20 & $2+$ \\
\hline$C-6$ & 60 & 104 & $75^{\dagger}$ & 0.25 & 83 \\
\hline G-6 & 60 & $22^{\dagger}$ & $24 \dagger$ & 0.25 & $8 \div$ \\
\hline Y-5 & 60 & $11 \dagger$ & $6^{+}$ & 0.50 & $24^{\dagger}$ \\
\hline mean $\pm \mathrm{SE}$ & - & $38 \pm 22 \%$ & $32 \pm 15 \%$ & $0.30 \pm 0.07$ & $30 \pm 18 \%$ \\
\hline B-6 & 30 & 110 & 86 & 0.37 & 末 \\
\hline C-6 & 30 & 81 & $44^{+}$ & 0.33 & $128^{+}$ \\
\hline G-6 & 30 & 99 & $72^{+}$ & 0.37 & 76 \\
\hline$Y-5$ & 30 & $11 \dagger$ & $16^{+}$ & 0.18 & $16^{\frac{1}{\dagger}}$ \\
\hline mean $\pm \mathrm{SE}$ & - & $75 \pm 22 \%$ & $55 \pm 16 \%$ & $0.31 \pm 0.50$ & $73 \pm 32 \%$ \\
\hline
\end{tabular}

*The duration of each CCK-8 intravenous infusion was 6 minutes.

$\dagger p<0.05$ when data points are compared with each animals own usual feeding pattern, by a one sample $t$-test. $\ddagger$ Data missing.

correlated with the size of the first meal: $t(16)=-4.2$, $p<0.001$, the duration of the first meal: $t(16)=-4.9$, $p<0.001$, and the duration of the first inter-meal interval: $t(15)=-2.5, p<0.03$, but had no significant relationship to total food intake for the test day nor for the subsequent day.

\section{CCK-8 Effect on Plasma Glucose Response to a Mixed Meal}

As can be seen in Fig. 2B and 2C for one representative animal, CCK -8 , infused at rates of $30-120 \mathrm{ng} / \mathrm{kg} / \mathrm{min}$, produced an obvious delay in the usual rise in plasma glucose and insulin in response to the oral ingestion of a mixed meal. These delays occurred in both free-feeding animals tested. Because CCK-8 administered during free feeding, as described above, truncated the initial meal, the attentuated glucose and insulin rises could have been associated with the reduced size of the meal load. In order to assess the effect of CCK on glucose and hormone responses to a mixed meal, independent of the effects on feeding behavior and the associated altered intake levels, the same diet, as was normally ingested orally, was infused directly into the stomach via an intragastric tube. A constant volume $(100 \mathrm{ml}$ or approximately $100 \mathrm{kcal}$ ) was infused at a rate of $10 \mathrm{ml} / \mathrm{min}$. Because some effects on free feeding occurred at CCK-8 infusion levels as low as $30 \mathrm{ng} / \mathrm{kg} / \mathrm{min}$, subsequent studies of the effects of CCK-8 on glucose and hormone responses to an intragastric mixed-meal load included a lower dose, 15 $\mathrm{ng} / \mathrm{kg} / \mathrm{min}$.

Under these conditions of controlled amount and rate of intragastric mixed-meal challenge, CCK-8 still produced apparent delays in the stimulated plasma glucose rise when compared to saline-infused controls (Fig. 3). During the first 40 minutes of the experiment, profile analysis revealed a significant CCK effect on the plasma glucose response to the intragastric infusion of a mixed meal. This effect was limited to comparisons between saline controls and CCK infused at the rates of 60 and $120 \mathrm{ng} / \mathrm{kg} / \mathrm{min}, \mathrm{F}(2,6)=5.7, p<0.05$. ANOVA and protected $t$-tests revealed that at the $10 \mathrm{~min}$ point plasma glucose response rise was lower for CCK-8 doses of 30,60 and $120 \mathrm{ng} / \mathrm{kg} / \mathrm{min}$ compared to saline controls, $\mathrm{F}(4,15)=4.3, \quad p<0.02$; however, protected $t$-tests demonstrated that only the difference between saline controls and $120 \mathrm{ng} / \mathrm{kg} / \mathrm{min}$ exceeded the Scheffe allowance. By the $20 \mathrm{~min}$ and $30 \mathrm{~min}$ time points glucose levels had returned toward saline control levels, and were significantly lower only at $20 \mathrm{~min}$ for the $120 \mathrm{ng} / \mathrm{kg} / \mathrm{min}$ dose $, t(8)=11.8, p<0.01$, and by $40 \mathrm{~min}$, no CCK-8 effect on plasma glucose was significant.

Overall plasma glucose response, based on integration of the glucose levels under the response curve for zero to 120 minutes, was not affected by the 15,30 or $60 \mathrm{ng} / \mathrm{kg} / \mathrm{min}$ doses (Fig. 4). Because of the delayed onset of the glucose response, and the lack of full return to baseline levels by the $120 \mathrm{~min}$ point at the $120 \mathrm{ng} / \mathrm{kg} / \mathrm{min}$ dose, the glucose response area was significantly smaller than normal saline controls, $t(10)=2.9, p<0.02$.

\section{CCK-8 Effect on Plasma Insulin Response to a Mixed Meal}

As with plasma glucose, and shown in Fig. 2C for one representative animal, there were delays in the plasma IRI response to intravenous $\mathrm{CCK}-8$ in free-feeding monkeys $(\mathrm{N}=2)$. When amount of the meal challenge and rate of delivery were controlled by intragastric infusion of the liquid diet, instead of oral feeding, a similar delay in plasma insulin response was observed (Fig. 5). Profile analyses revealed a CCK effect on insulin response rise which approached significance when saline controls and CCK-8 infused at the rates of 60 and $120 \mathrm{ng} / \mathrm{kg} / \mathrm{min}$ were compared, $F(2,6)=4.5$, $p=<0.06$. ANOVA demonstrated these differences occurred 


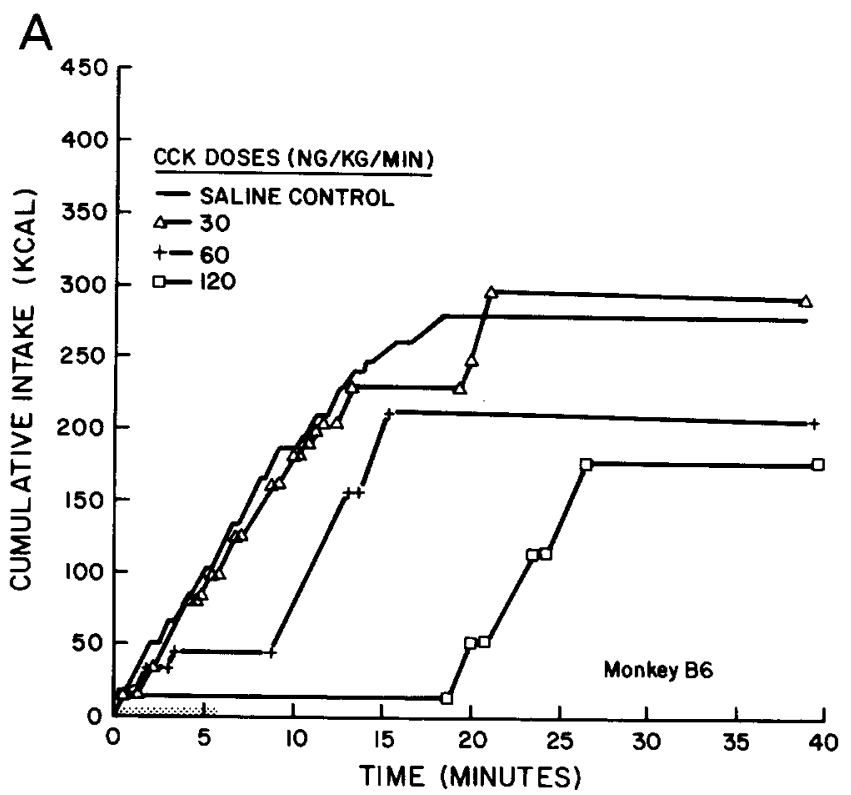

FIG. 2A. Cumulative diet intake (kcal) during the first 40 min of free feeding following an overnight fast for a single representative animal (Monkey B-6). Varying doses of CCK-8 were infused during the first 6 min only. The period of infusion is shown by the stippled area.

B

\section{GLUCOSE RESPONSE RISE TO FREE FEEDING AND IV CCK-8}

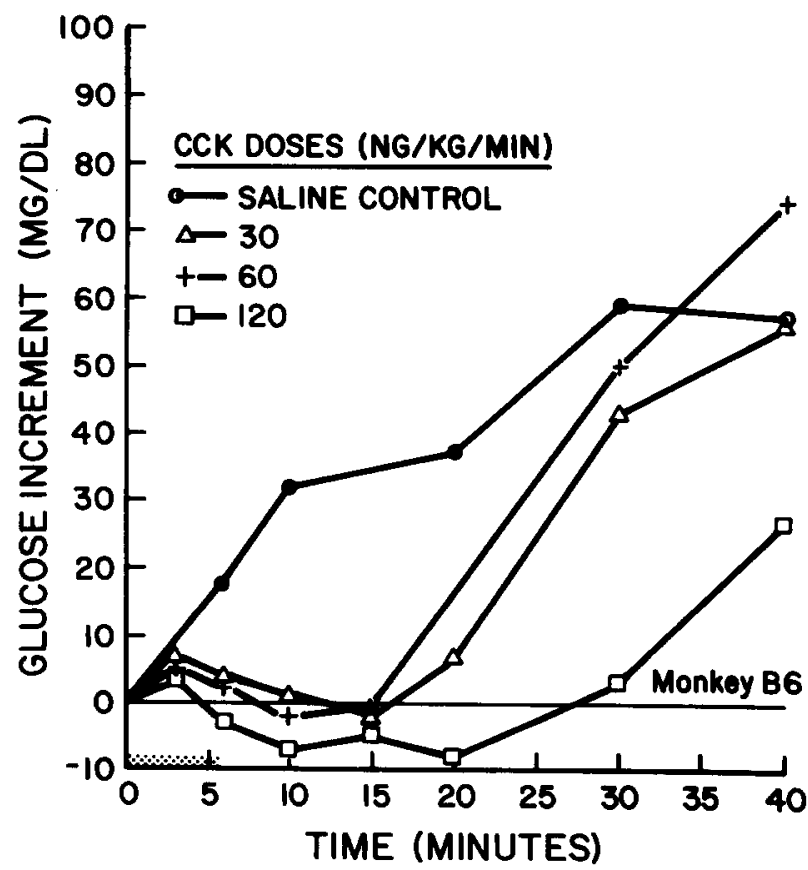

FIG. 2B. Plasma glucose response (rise above baseline level) to 6 min infusions of saline (control) and varying doses of CCK-8 during the free feeding experiment shown in Fig. $2 \mathrm{~A}$ for a single representative animal (Monkey B-6). The period of infusion is shown by the stippled area.
C

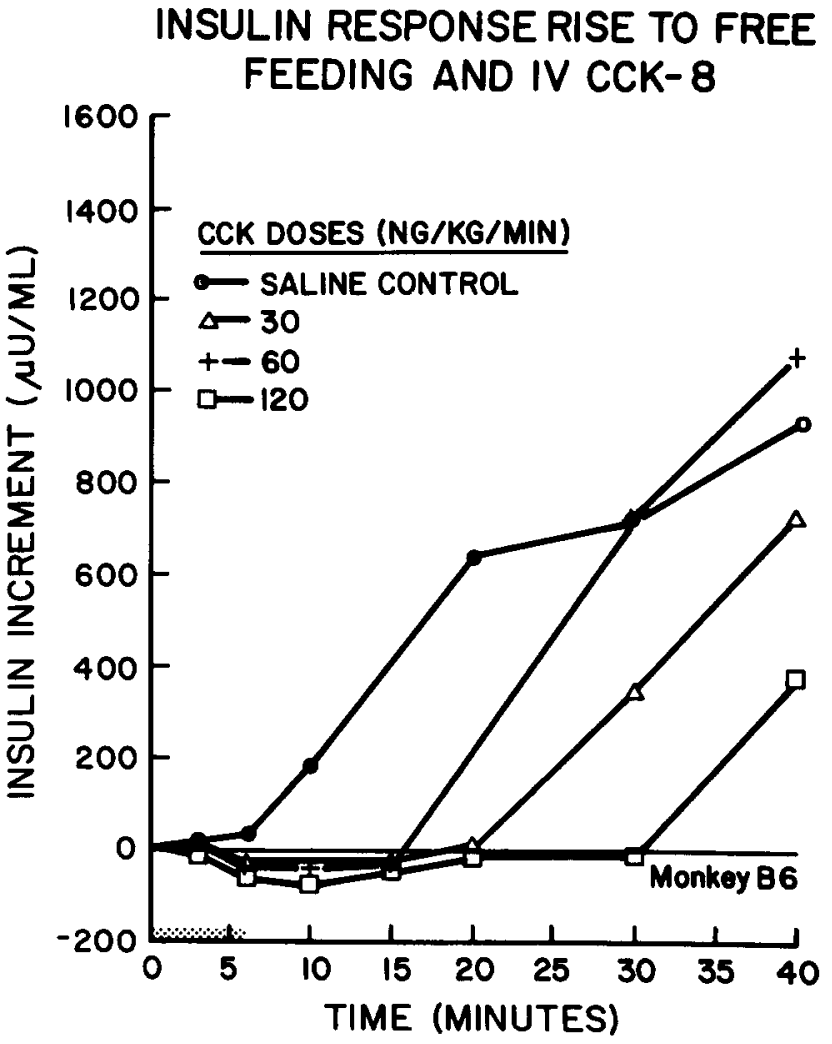

FIG. 2C. Plasma insulin response (rise above baseline level) to $6 \mathrm{~min}$ infusions of saline (control) or varying doses of CCK-8 during the free feeding experiment shown in Fig. $2 \mathrm{~A}$ for a single representative animal (Monkey B-6). The period of infusion is shown by the stippled area. 


\section{GLUCOSE INCREMENT RESPONSE TO IG MIXED MEAL AND CCK}

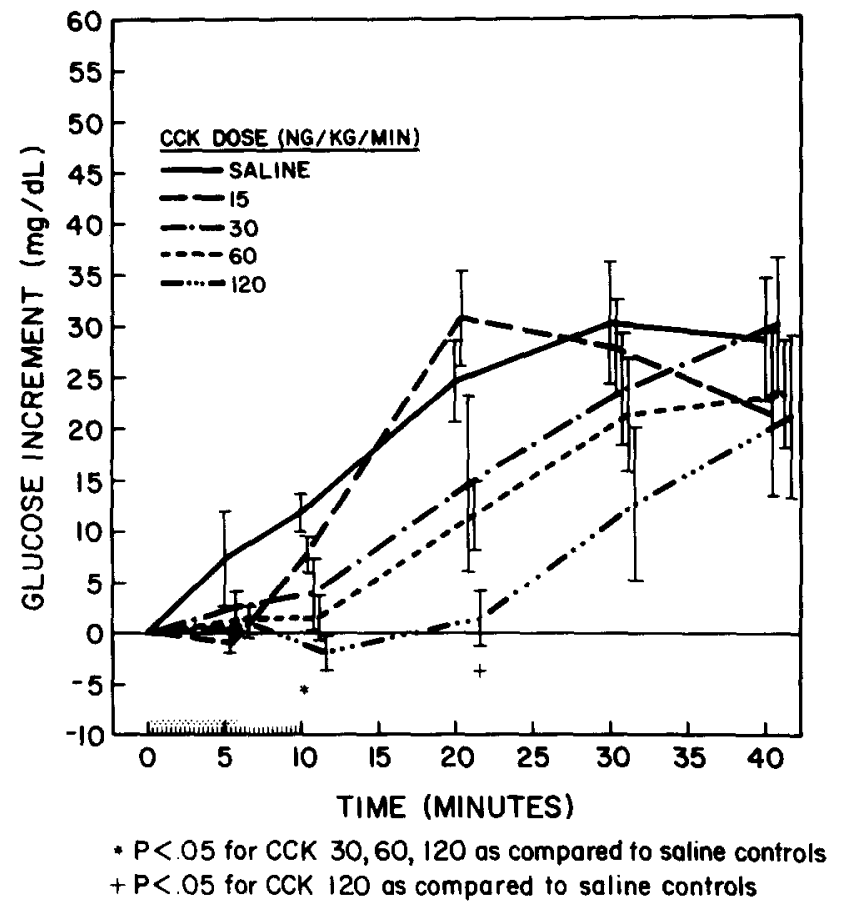

FIG. 3. Plasma glucose change from baseline (overnight fasted) levels during the following $10 \mathrm{~min}$ intragastric infusion of a mixed meal. CCK-8 was infused intravenously over a $6 \mathrm{~min}$ period at four dose levels ( $N=4$ monkeys). The period of CCK infusion is indicated by the stippled area, and the period of the intragastric infusion by the dashed area at the bottom.

at the 10 and 20 min time points; but when all possible pair combinations were contrasted only the difference between saline controls and CCK infused at $120 \mathrm{ng} / \mathrm{kg} / \mathrm{min}$ at the 20 min point was significant, $t(8)=6.5, p<0.03$. This difference did not, however, exceed the Scheffe allowance at the 0.95 level. One change observed in the IRI response did not parallel the changes observed in plasma glucose: following CCK infused at the highest dose $(120 \mathrm{ng} / \mathrm{kg} / \mathrm{min})$ at the $10 \mathrm{~min}$ time period, the mean IRI level of $48.1 \mu \mathrm{U} / \mathrm{ml}$ was significantly lower than mean basal IRI levels of $67 \mu \mathrm{U} / \mathrm{ml}, t(8)=-2.4$, $p<0.05$. By contrast, the $15 \mathrm{ng} / \mathrm{kg} / \mathrm{min}$ dose produced a slight (non-significant) delay in rise of plasma insulin level at the 5 min point, and possibly enhanced the plasma insulin responses at 10, 20 and $30 \mathrm{~min}$ compared to normal saline controls (Fig. 5).

As with glucose, the total insulin response (area under the curve-zero to $120 \mathrm{~min}$ ) was reduced only by the 120 $\mathrm{ng} / \mathrm{kg} / \mathrm{min}$ dose, $t(10)=2.6, p<0.05$, and this appeared to be an artifact of the duration of sampling and the lack of samples between the 60 and 120 min time points, when the delayed peak insulin values were undoubtedly reached (Fig. 4).

The ratio of the standardized values for plasma insulin and plasma glucose showed no significant differences during the $120 \mathrm{~min}$ for any CCK-8 dose compared to a normal saline infusion. CCK-8 clearly did not disrupt the close association of plasma glucose and insulin in response to a mixed meal; there was even a slight tendency for CCK-8 at $120 \mathrm{ng} / \mathrm{kg} / \mathrm{min}$ to strengthen the insulin-glucose relationship, relative to

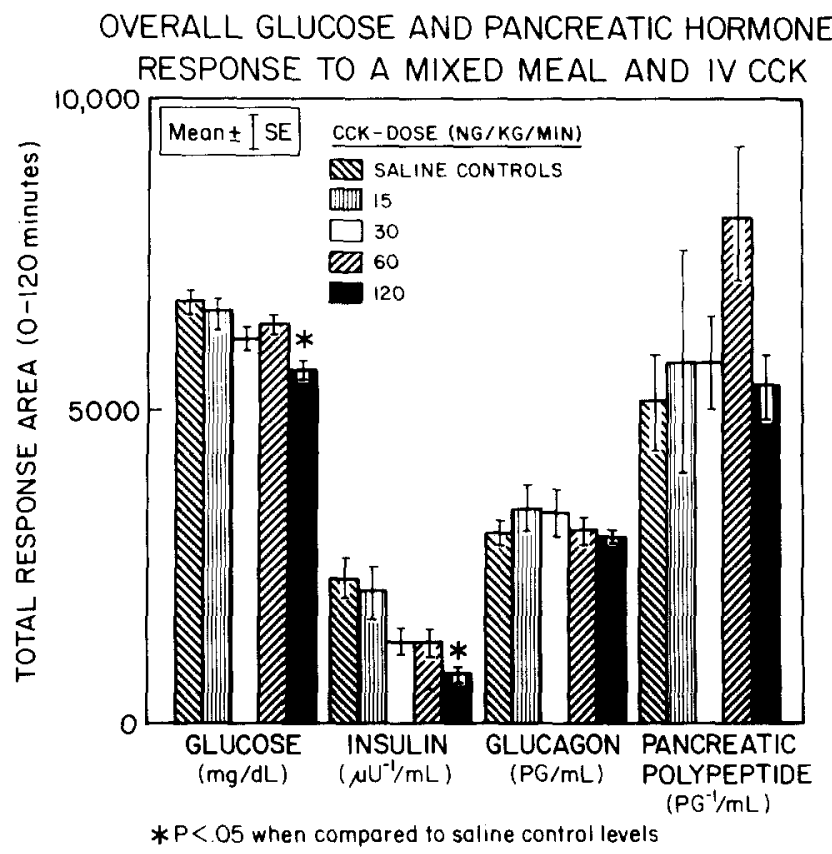

FIG. 4. Total integrated response area of plasma glucose, insulin, glucagon and pancreatic polypeptide during the first 120 min following simultaneous initiation of $10 \mathrm{~min}$ intragastric mixed meal infusions and $6 \mathrm{~min}$ intravenous CCK-8 infusions at four dose levels compared to saline control infusions ( $N=5$ monkeys).

INSULIN INCREMENT IN RESPONSE TO IG MIXED MEAL AND CCK

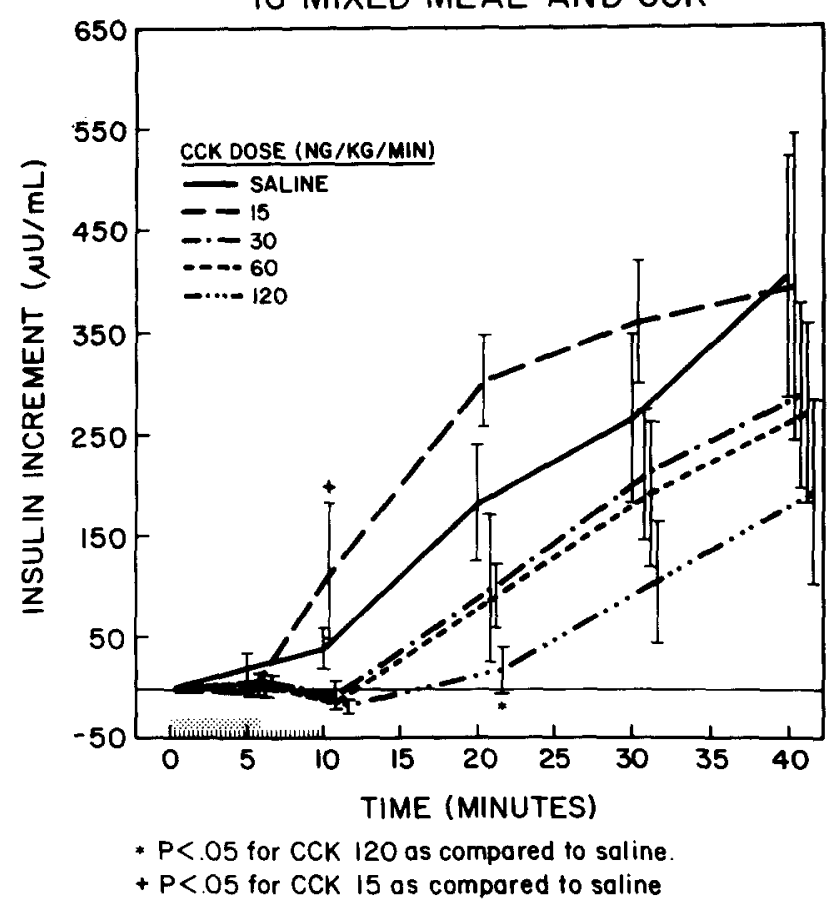

FIG. 5. Plasma insulin changes from baseline (overnight fasted) levels following $10 \mathrm{~min}$ intragastric infusion of a mixed meal and simultaneous 6 min intravenous infusion of four dose levels of CCK-8. The periods of the respective infusions are shown by stippled and dashed areas ( $N=4$ monkeys). 
GLUCAGON INCREMENT RESPONSE TO IG MIXED MEAL AND CCK

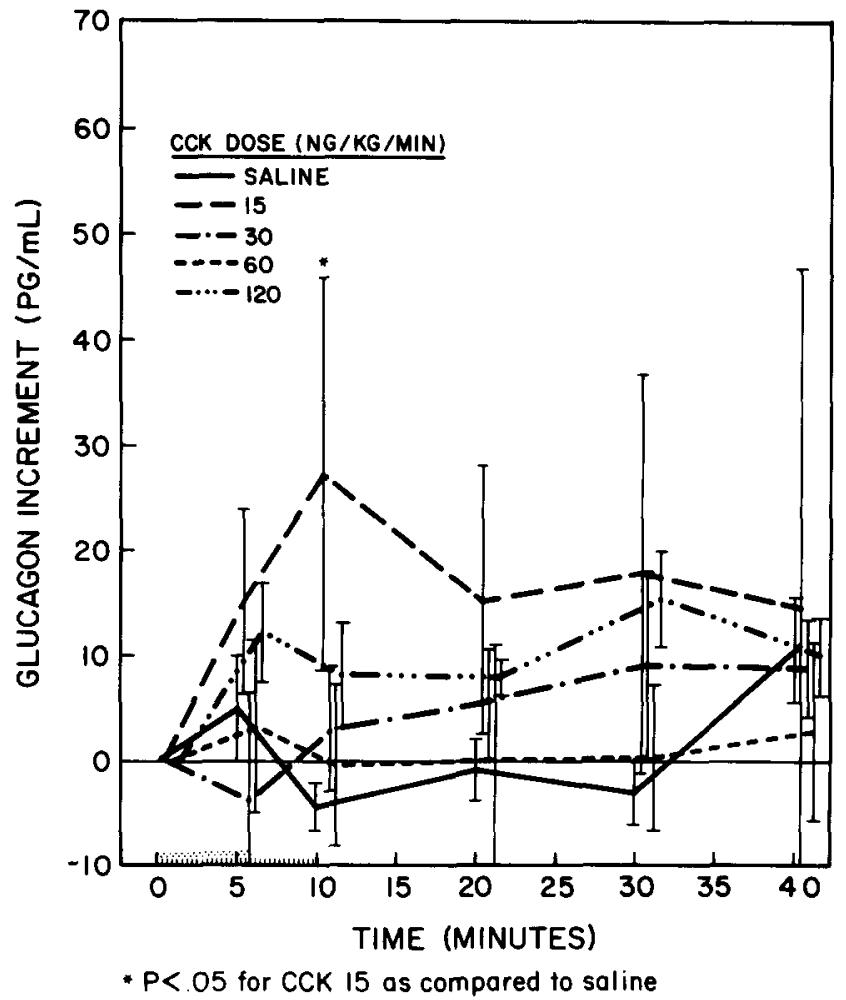

FIG. 6. Plasma glucagon changes from baseline levels following 10 min intragastric infusion of a mixed meal with simultaneous intravenous infusion of four different doses of CCK-8 during the first 6 min $(\mathrm{N}=4$ monkeys). The periods of the respective infusions are shown as in Fig. 5.

ratios at $15 \mathrm{ng} / \mathrm{kg} / \mathrm{min}$ or during saline controls. As shown in Table 2, plasma glucose and insulin levels were highly correlated at all CCK-8 dose levels.

\section{CCK-8 Effect on Plasma Glucagon and Pancreatic} Polypeptide Responses to a Mixed Meal

A six min intravenous infusion of CCK-8 had no consistent effect on the response of either plasma glucagon or pancreatic polypeptide to intragastric infusion of a mixed meal (Figs. 6 and 7). While levels of both hormones were generally higher during CCK-8 infusions, no consistent dose-related effects were observed. Only the $15 \mathrm{ng} / \mathrm{kg} / \mathrm{min}$ dose produced a significant increase in plasma glucagon-and then only at the $10 \mathrm{~min}$ time point. Large variability was observed in these hormone responses.

\section{Non-Feeding Behavior: Effect of $\mathrm{CCK}-8$}

Clinical signs of illness during the CCK-8 infusions were not observed. The monkeys showed no retching, vomiting, agitation, unusual drowsiness, or change in stool number or consistency. Maximal likelihood tests of independence demonstrated no CCK-8 dependent differences in behavior between the oral free feeding and the intragastric diet infusion conditions, except for the $120 \mathrm{ng} / \mathrm{kg} / \mathrm{min}$ dose: the monkeys engaged in significantly more resting behavior at this CCK dose level under the free feeding condition than under

\section{PANCREATIC POLYPEPTIDE (PP) INCREMENT} RESPONSE TO IG MIXED MEAL AND CCK

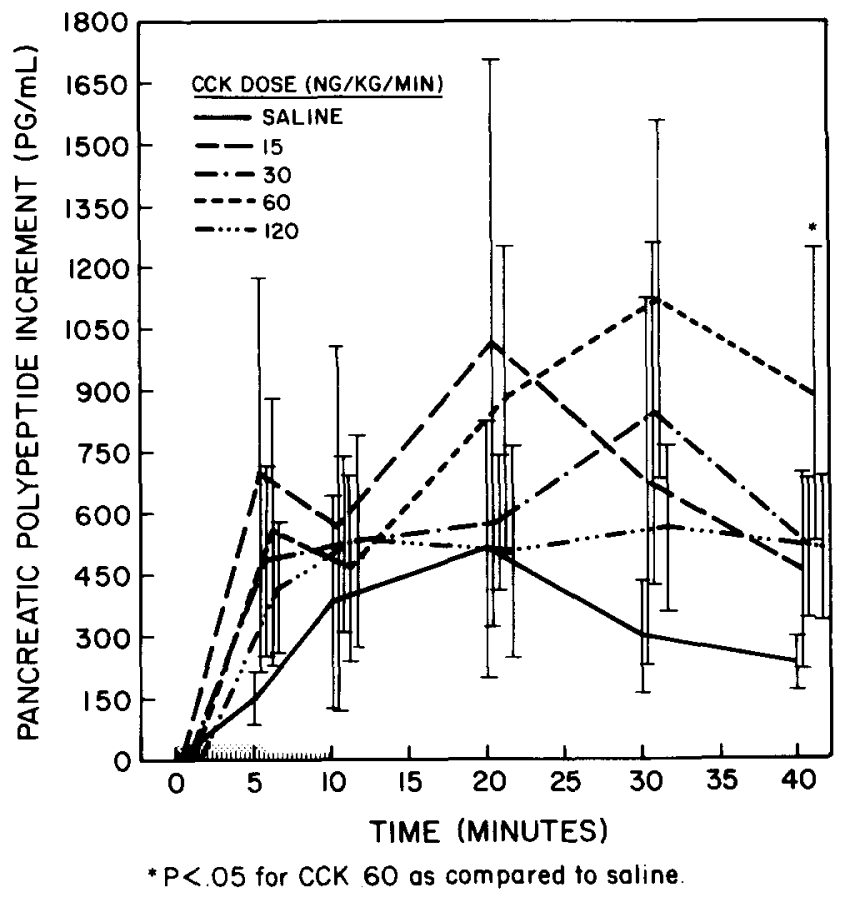

FIG. 7. Plasma pancreatic polypeptide changes from baseline levels during and following 10 min intragastric mixed meal infusion and 6 min intravenous infusion of four doses of CCK-8 compared to saline infusions $(\mathrm{N}=4$ monkeys). Period of the respective infusions are shown as in Fig. 5.

TABLE 2

INTERACTION BETWEEN PLASMA GLUCOSE AND INSULIN RESPONSES TO AN IG INFUSION OF A MIXED MEAL AND SIX MINUTE IV INFUSIONS OF CCK- ${ }^{*}$

\begin{tabular}{lccccc}
\hline & \multicolumn{5}{c}{ CCK Dose (ng/kg/min) } \\
Monkey & saline & 15 & 30 & 60 & 120 \\
\hline C-6 & $.82 \dagger$ & $.74 \dagger$ & $.93 \pi$ & $.90 \ddagger$ & $.79 \ddagger$ \\
V-5 & $.76 \dagger$ & - & $.97 \uparrow$ & $.97 \uparrow$ & $.96 \AA$ \\
Y-5 & $.77 \dagger$ & $.85 \ddagger$ & $.94 \S$ & $.94 \ddagger$ & $.99 \uparrow$ \\
G-6 & $.90 \ddagger$ & $.87 \ddagger$ & $.97 \pi$ & $.89 \ddagger$ & $.93 \ddagger$ \\
Mean & $.81 \dagger$ & $.82 \ddagger$ & $.95 \uparrow$ & $.92 \ddagger$ & $.92 \ddagger$ \\
\hline
\end{tabular}

*All interactions are expressed as a product-moment correlation coefficient.

$\dagger p<0.05, \neq p<0.01, \S p<0.001$ and $\Phi p<0.0001$ when the strength of the product-moment correlation coefficient is tested by the $t$-statistic.

the intragastric diet infusion condition. Monkeys were, in fact, generally more active during the intragastric infusion than while free feeding. There were no other CCKassociated effects on non-ingestive behavior during the 45 minute observation period. 


\section{DISCUSSION}

Intravenous administration of CCK- 8 over a 6 min period coincident with the initiation of free feeding following an overnight fast resulted in a dose-related cessation of the ongoing meal. The threshold for this effect ranged from 30 to $120 \mathrm{ng} / \mathrm{kg} / \mathrm{min}$ across monkeys, and was apparent within 2 min of the start of the infusion. In a similar protocol using solid food ingestion by monkeys as the test parameter, Gibbs, Falasco, and McHugh [6] also reported significant suppression of feeding (a $66 \%$ decrease) using a dose of CCK slightly larger than our largest dose. Their assessment of feeding behavior was, however, limited to total amount of food taken at the 15, 30,60 and 120 min time points and early effects would therefore, have been obscured. In all of the monkeys tested in the present study some recovery of feeding has already occurred by the 15 min time point and only the period between 0 and 20 minutes provided sensitive information about the threshold effects of CCK-8.

When a dose of CCK-8, sufficient to transiently terminate feeding, was used we observed no effect on feeding rate either prior to cessation of feeding, or at the time feeding resumed. Anika, Houpt and Houpt [1] reported similar effects on feeding behavior in pigs, however, their doses were probably higher than those we have found to be effective in monkeys.

Our data indicated an extremely rapid and transient time course for the effect of intravenous CCK-8, and support the Gibbs and associated [6] observation that most effects do not extend beyond $15 \mathrm{~min}$ after CCK infusion in monkeys. The present data contrast, however, with their finding that monkeys do not compensate for early decreases in food intake following short term CCK administration [6]. However, it should be noted that Gibbs, Falasco and McHugh infused impure CCK, in contrast to the synthetic CCK-8 used in this study. Since practical clinical value may depend upon a residual effect of CCK-8, we looked carefully at the entire $8 \mathrm{hr}$ feeding pattern rates, and intake amounts across time, and total intake on the test day compared to preceding and succeeding days. By $3 \mathrm{hrs}$ post CCK-8 infusion total cumulative intake for the day had returned to control levels indicating that the lean monkeys compensated well for the early and transient decrease in food intake. Neither the total intake for the day, nor the intake on the following day showed any effect of the CCK dose. This contrasts with the report of a residual CCK effect observed over days in man [11], but is similar to Hsaiso's finding of an absence of prolonged effects of CCK in rats [9].

This rapid and transient effect of CCK- 8 on feeding behavior, combined with our finding of early compensation for the short term depression of food intake probably accounts for the lack of effect of CCK-8 (doses ranging from $1 \mu \mathrm{g} / \mathrm{kg} / 5$ $\min$ to $4 \mu \mathrm{g} / \mathrm{kg} / 5 \mathrm{~min}$ ), on food intake in the baboon following an overnight fast [26]. Food intake of the baboon was measured over $30 \mathrm{~min}$ after the end of a $5 \mathrm{~min}$ CCK infusion. If baboons are similar to rhesus monkeys within 10 to 20 min following the CCK infusion food intake would have been reinitiated, and since normal meal duration probably approximates $10 \mathrm{~min}$, it is not surprising that no reliable effect of CCK on food intake was observed at the $30 \mathrm{~min}$ time point [26].

As we have found in monkeys, Hsiao and others [9] and Mueller and Hsiao [21], using similar protocols in rats, showed a significant CCK effect on truncating the size of a meal, and no effect on rate of feeding. In both the monkeys and rats, the shortened IMI after the truncated first meal probably related to the reduced meal size, and not to any early disinhibition of feeding.

As described by Gibbs, Falasco and McHugh [6] and Falasco, Smith and Gibbs [3], monkeys showed no signs of illness or distress during CCK infusions. Specific observation of types of behavior changes elicited during the first 30 minutes showed the same increase in resting behavior as was described by Falasco, Smith and Gibbs [3]. Whether the decrease in food intake with CCK-8 is related to any mild sensations of malaise or other non-specific effects of CCK is difficult to ascertain.

Behavioral monitoring of the monkeys suggested that under the free feeding conditions at the largest dose, $\mathrm{CCK}-8$ produced significantly more resting behavior compared to saline. That this might be related to the combination of CCK plus pregastric factors was suggested by the finding that behavior during the CCK plus intragastric meal infusion did not differ from saline plus the intragastric meal. Thus some pregastric stimuli may be necessary for the CCK-associated satiety behavior to occur. Antin, Gibbs and Smith [2], and Kraly and others [12] using sham-fed rats found a synergism between CCK and pregastric factors, suggesting complementary roles in producing satiety behavior.

In the present studies, the rise of plasma glucose during the course of ingestion or infusion of a mixed meal was significantly delayed during CCK-8 infusions, but total glucose response in the post infusion period was unaffected by the preceding CCK. Using intraperitoneal CCK-8 doses five times higher than our largest cumulative intravenous dose, McLaughlin and Baile [18] found decreased glucose response to a meal for at least 60 minutes in obese and lean rats. With doses 1.5 to six times greater than ours, Woods and Stein [26] found that CCK-8 reliably attenuated the glucose response to the subsequent meal at the time point 30 min after the start of the meal. The highest dose used for our monkeys also showed significant suppression of the glucose response. Our data show, however, that this is a dose dependent delay, and not an attenuation of response since the integrated glucose area or incremental area calculated to 120 min was normal except for the highest dose where glucose had not yet returned to basal levels at the $120 \mathrm{~min}$ time point. In light of Moran and McHugh's demonstration (using approximately $1 / 10$ of our CCK-8 dose) that CCK-8 consistently produced rapid and transient delay or slowing of gastric emptying [20], a possible explanation is that the doserelated delay in plasma glucose response to a mixed meal is a direct function of the slowed gastric emptying.

The doses of CCK used in these experiments were considerably larger than those producing contraction of the gall bladder in humans and baboons [13], and the observed effects of CCK-8 are, therefore, possibly pharmacological. Although the physiological role of CCK in appetite regulation is open to question at least until postprandial plasma CCK levels can be ascertained and its release from intestinal sites selectively inhibited, it may very well act in concert with other satiety-affecting factors. Its apparent short time course of action, the occurrence of gastrointestinal side effects at high doses, the decreased sensitivity of obese Zucker rats to its satiety effect [16] and possible habituation over time [19] may limit the therapeutic potential as an appetite suppressant.

Our studies of intravenous CCK-8 effects on plasma insulin and glucagon levels in awake, mixed meal-stimulated monkeys differs from the effects observed by Rouiller and 
associates [23] in anesthetized dogs. Infusing CCK-8 over a longer period, but at a dose rate equivalent to $30 \mathrm{ng} / \mathrm{kg} / \mathrm{min}$, they reported significant and rapid (within $5 \mathrm{~min}$ ) rises in plasma insulin and glucagon, with incremental values significantly higher than for saline controls [23]. One might expect that the superimposing of a mixed meal on top of the CCK-8 infusion would produce at least as rapid and significant a rise in insulin as was observed for CCK-8 alone in the anesthetized dog. This was clearly not true for our monkeys where the rise in plasma insulin was significantly delayed (more than 15 minutes) by the CCK-8 infusion at all doses larger than $15 \mathrm{ng} / \mathrm{kg} / \mathrm{min}$.

Frame and associates [5] have proposed that CCK stimulates the "quick release" pool of insulin, however, the doses of CCK used in our monkeys failed to produce this effect. Instead, our data are in accord with the findings of Jensen and others [10] using the isolated perfused porcine pancreas, in that CCK-8 did not significantly stimulate the secretion of insulin.

CCK may stimulate glucagon release, but the effect is small. Neither the mixed meal nor the intravenous CCK produced a significant change in plasma glucagon levels, although comparisons to saline controls suggested some variable increases. This again, contrasts with the immediate large stimulation of glucagon release during CCK-8 infusions in the anesthetized dog [23]. Species and protocol differences, including differences in time points for response measurement, the type and molecular form of the CCK used and CCK dose all confound the interpretation of these diverse findings.

Lonovics et al. [15] have shown in dogs that CCK-8 produced an effect on plasma levels of pancreatic polypeptide similar to the effect of ingestion of a mixed meal, and have suggested that CCK may be an important mediator of PP release. Our results suggest that exogenous CCK may produce a small additive effect on the PP response to a mixed meal. Although variability prevented the finding of significant enhancement by CCK, PP was consistently higher during CCK infusion with a mixed meal when compared to saline infusion. Even where there was a probable delay in test meal absorption as indicated by the delay in plasma glucose rise, pancreatic polypeptide showed an early rise and remained above control levels. There was, however, no observable dose-response relationship.

In summary, intravenous CCK-8 infused during the first 6 min of an oral or intragastric mixed meal in monkeys produced significant dose related inhibition of feeding behavior, delayed but did not otherwise alter the plasma glucose and insulin responses, variably enhanced (not significantly) the release of glucagon and pancreatic polypeptide, and had no effects extending beyond 3 hours. Despite the consistent delays in plasma glucose and insulin responses to a mixed meal at all CCK-8 infusion rates greater than $15 \mathrm{ng} / \mathrm{kg} / \mathrm{min}$, the minimal effective dose for feeding inhibition varied from 30 to $120 \mathrm{ng} / \mathrm{kg} / \mathrm{min}$ in the same monkeys. This finding suggests that the CCK-induced delays in plasma insulin and glucose response are not sufficient to the feeding effect. Clearly, insulin did not mediate the CCK-8-induced decrement in food intake, although delay of the early insulin response to a meal may be necessary to the effect on feeding. The usual close relationship between plasma glucose and insulin levels in response to a mixed meal was not significantly changed by the administration of intravenous CCK.

\section{ACKNOWLEDGEMENTS}

The authors acknowledge the support and assistance of Dr. K-L. C. Jen. Excellent technical assistance was provided by S. Gray, L. Speegle and J. Schielke.

\section{REFERENCES}

1. Anika, S. M., T. R. Houpt and K. A. Houpt. Cholecystokinin and satiety in pigs. Am ll Physiol 9: R310-R318, 1981.

2. Antin, J., J. Gibbs and G. P. Smith. Cholecystokinin interacts with pregastric food stimulation to elicit satiety in the rat. Physiol Behav 20: 67-70, 1978.

3. Falasco, J. D., G. P. Smith and J. Gibbs. Cholecystokinin suppresses sham feeding in the rhesus monkey. Physiol Behav 23: 887-890, 1979.

4. Floyd, J. C., Jr., S. S. Fajans, S. Pek and R. E. Chance. A newly recognized pancreatic polypeptide: Plasma levels in health and disease. Recent Prog Horm Res 33: 519-570, 1977.

5. Frame, C. M., M. B. Davidson and R. A. L. Sturdevant. Effects of the octapeptide of cholecystokinin on insulin and glucagon secretion in the dog. Endocrinology 97: 533-549, 1975.

6. Gibbs, J., J. D. Falasco and P. R. McHugh. Cholecystokinindecreased food intake in rhesus monkeys. Am J Physiol 230: $15-18,1976$

7. Harris, R. J. A Primer of Multivariate Statistics. New York: Academic Press, 1975, pp. 80-84.

8. Hayashi, M., J. C. Floyd, Jr., S. Pek and S. S. Fajans. Insulin, proinsulin, glucagon and gastrin in pancreatic tumors and in plasma of patients with organic hyperinsulinism. $J$ Clin Endocrinol Metab 44: 681-694, 1977.

9. Hsiao, S., C. H. Wang and T. Schallert. Cholecystokinin, meal pattern, and the intermeal interval: Can eating be stopped before it starts? Physiol Behav 23: 909-914, 1979.
10. Jensen, S. L., J. F. Rehfeld, J. J. Holst, O. V. Nielsen, J. Fahrenkrug and O. B. S. De Muckadell. Secretory effects of cholecystokinins on the isolated perfused porcine pancreas. Acta Physiol Scand 111: 225-231, 1981.

11. Kissileff, H. R., F. X. Pi-Sunyer, J. T. Thornton and G. P. Smith. C-terminal octapeptide of cholecystokinin decreases food intake in man. Am J Clin Nutr 34: 154-160, 1981.

12. Kraly, F. S., W. J. Carty, S. Resnick and G. P. Smith. Effect of cholecystokinin on meal size and intermeal interval in the sham-feeding rat. J Comp Physiol Psychol 92: 696-707, 1978.

13. LaMorte, W. W., J. M. Gaca, W. E. Wise, D. H. Birkett and L. F. Williams, Jr. Choledochal sphincter relaxation in response to histamine in the primate. J Surg Res 28: 373-378, 1980.

14. Le Magnen, J. The metabolic basis of dual periodicity of feeding in rats. Behav Brain Sci 4: 561-607, 1981.

15. Lonovics, J., S. Guzman, P. Devitt, K. E. Hejtmanick, R. L. Suddith, P. L. Rayford and J. C. Thompson. Release of pancreatic polypeptide in humans by infusion of cholecystokinin. Gastroenterology 79: 817-822, 1980.

16. McLaughlin, C. L. and C. A. Baile. Decreased sensitivity of Zucker obese rats to the putative satiety agent cholecystokinin. Physiol Behav 25: 543-548, 1980.

17. McLaughlin, C. L. and C. A. Baile. Obese mice and the satiety effects of cholecystokinin, bombesin and pancreatic polypeptide. Physiol Behav 26: 433-437, 1981.

18. McLaughlin, C. L. and C. A. Baile. Serum insulin, glucose and triglyceride responses of Zucker obese and lean rats to cholecystokinin. Physiol Behav 26: 995-999, 1981. 
19. Mineka, S. and C. T. Snowdon. Inconsistency and possible habituation of CCK-induced satiety. Physiol Behav 21: 65-72, 1978.

20. Moran, T. H. and P. R. McHugh. Cholecystokinin suppresses food intake by inhibiting gastric emptying. Am J Physiol 242: R491-R497, 1982.

21. Mueller, K. and S. Hsiao. Consistency of cholecystokinin satiety effect across deprivation levels and motivational states. Physiol Behav 22: 809-815, 1979.

22. Pek, S. Glucagon and insulin. In: Nuclear Medicine: Endocrinology, edited by B. Rothfield. Philadelphia: J. B. Lippincott, 1978 , pp. $122-140$.
23. Rouiller, D., V. Schusdziarra, V. Harris and R. H. Unger. Release of pancreatic and gastric somatostatin-like immunoreactivity in response to the octapeptide of cholecystokinin, secretin, gastric inhibitory polypeptide, and gastrin-17 in dogs. Endocrinology 107: 524-529, 1980.

24. Slein, M. W. D-Glucose determinations with hexokinase and glucose-6-phosphate dehydrogenase. In: Methods of Enzymatic Analysis, edited by H. U. Bergmeyer. New York: Academic Press, 1971, pp. 117-123.

25. Walike (Hansen), B. C., D. J. Campbell and R. A. Hillman. A liquid diet feeder for monkeys. J Appl Physiol 31: 946-947, 1971.

26. Woods, S. C. and L. H. Stein. Intravenous cholecystokinin (CCK) blocks glucose and insulin responses to meals in free feeding baboons. Diabetes 31: Suppl 2, 121, 1982. 